\title{
The effects of gamma-radiation on the properties of Brillouin scattering in standard Ge-doped optical fibres
}

\author{
Dario Alasia $^{1}$, Alberto Fernandez Fernandez ${ }^{2}$, Laura Abrardi ${ }^{1}$, \\ Benôit Brichard $^{2}$ and Luc Thévenaz ${ }^{1}$ \\ ${ }^{1}$ EPFL, Ecole Polytechnique Fédérale de Lausanne, Nanophotonics and Metrology \\ Laboratory, STI-NAM Station 11, CH-1015 Lausanne, Switzerland \\ ${ }^{2}$ SCK•CEN, Belgian Nuclear Research Centre, Boeretang 200, B-2400 Mol, \\ Belgium \\ E-mail: dario.alasia@epfl.ch and luc.thevenaz@epfl.ch
}

Received 13 July 2005, in final form 28 October 2005

Published 7 April 2006

Online at stacks.iop.org/MST/17/1091

\begin{abstract}
We have experimentally studied the effects of gamma-radiation up to very high total doses on the physical properties of Brillouin scattering in standard commercially available optical fibres. A frequency variation of about $5 \mathrm{MHz}$ for both Brillouin frequency and linewidth has been measured at the total dose of about $10 \mathrm{MGy}$. The radiation-induced shift has a negligible practical impact and makes Brillouin scattering very immune to radiation, so that distributed sensors based on this interaction exhibit an interesting potential for use in nuclear facilities.
\end{abstract}

Keywords: radiation effects, ionizing radiation, Brillouin scattering, distributed measurements, fibre optics sensors

(Some figures in this article are in colour only in the electronic version)

\section{Introduction}

Fibre optics sensing technology is under evaluation by the nuclear industry since it may bring promising alternatives to classical measurement techniques in harsh nuclear environments [1-3]. Distributed fibre optics sensing technologies would allow structural integrity monitoring of reactor containment buildings, nuclear waste repository survey and remote safety control of nuclear installations with significant benefits over conventional electronic instrumentation.

However, it is well known that the exposure of optical fibres to ionizing radiation results in a wavelength-dependent attenuation penalty. This effect limits the radiationacceptance level of intensity-based fibre optic sensors in nuclear environments and results in some radiation-induced errors which restrict the applicable area of such fibre sensors. The potentialities of Raman distributed temperature sensors have already been studied in radiation environments [4-6]. Special correction techniques for radiation-induced losses need to be used because differential radiation-induced attenuation for the Stokes and anti-Stokes lines could cause incorrect temperature measurements. In contrast, the narrow wavelength encoding of the sensing information helps to avoid the influence of the broadband radiation-induced loss, as already shown for fibre Bragg grating sensors [7].

Distributed sensors based on stimulated Brillouin scattering have an interesting potential for distributed strain and temperature monitoring in the nuclear industry. Since the sensing information is frequency-encoded, hence potentially radiation tolerant, it was interesting to study the radiation effect on the Brillouin shift for application in ionizing environments.

In this paper, we present novel results on the properties of the Brillouin gain spectrum under high gamma irradiation in a standard optical fibre up to a total gamma dose of about 10 MGy.

\section{Description of experimental conditions}

\subsection{Sample preparation}

We investigated the effects of ionizing radiation on the characteristics of the Brillouin gain spectrum in standard 


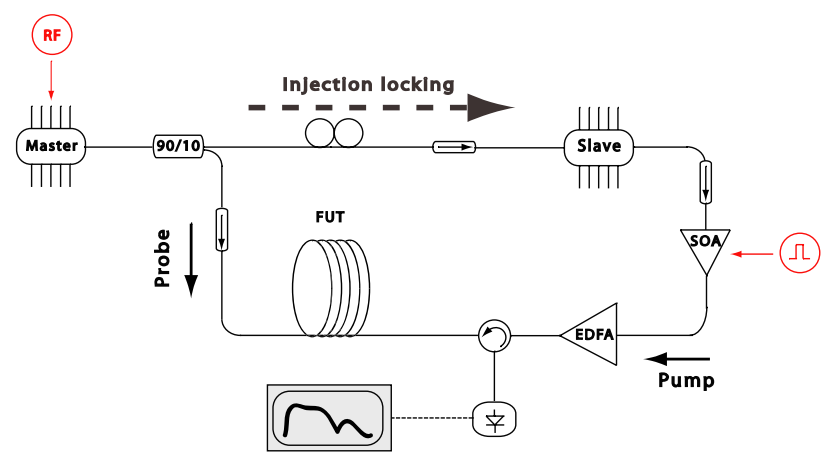

Figure 1. Schematic diagram of the injection-locking based configuration for Brillouin sensing. Master, slave: distributed feedback lasers; SOA: semiconductor optical amplifier; EDFA: erbium-doped fibre amplifier; FUT: fibre under test.

Table 1. A radiation dose experienced by the five samples of Lucent Allwave $^{\mathrm{TM}}$ single-mode fibres before measuring their Brillouin gain spectrum.

\begin{tabular}{lll}
\hline Fibre sample & Length $(\mathrm{m})$ & Dose (MGy) \\
\hline Reference & 50 & 0 \\
1 & 50 & 0.33 \\
2 & 50 & 0.97 \\
3 & 50 & 4.70 \\
4 & 50 & 9.90 \\
\hline
\end{tabular}

Ge-doped telecom single mode fibres (Lucent Allwave ${ }^{\mathrm{TM}}$ ). During this irradiation campaign, four fibre samples, cleaved to a common $50 \mathrm{~m}$ length, were irradiated off-line in the underwater gamma irradiation facility 'BRIGITTE' of $\mathrm{SCK} \bullet \mathrm{CEN}$, Belgium [8]. The test chamber consists of a pressurized canister, in which the samples are placed, which is then lifted down into the irradiation facility such that the samples are surrounded by ten ${ }^{60} \mathrm{Co}$ sources. In this configuration the fibres have been irradiated at the same dose rate of $27 \mathrm{kGy} \mathrm{h}^{-1}$ but up to different total doses.

The measured total dose absorbed by each fibre is listed in table 1 .

\subsection{Experimental set-up}

In order to evaluate the effect of radiation on the properties of Brillouin scattering a distributed measurement technique (BOTDA) has been used. The distributed measurement made it possible to discriminate the Brillouin characteristics of the irradiated fibre segment from those of the lead fibres.

The experimental configuration is schematically shown in figure 1. Two distributed feedback lasers-called respectively master and slave-generate two counterpropagating lightwaves at $1550 \mathrm{~nm}$-called respectively probe and pump - whose frequency separation must be kept constant and close to the Brillouin shift $\left(v_{\mathrm{B}}\right)$ of the fibre under test. An efficient technique to obtain these waves is by modulating [9] the master laser at the Brillouin frequency $(10-11 \mathrm{GHz})$ and by injection-locking the slave laser on one of the sidebands of the master [10]. This is achieved by coupling a portion of the light of the master into the cavity of the slave [11]. Using this simple and convenient technique the optical frequency difference

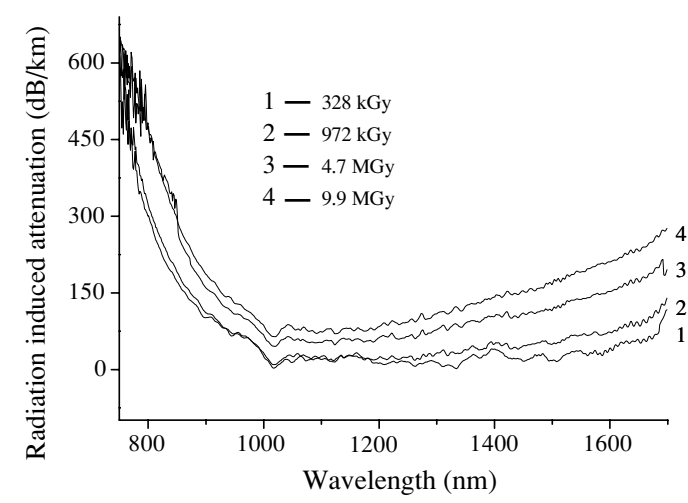

Figure 2. Spectral response of the radiation-induced attenuation. Curves are normalized with respect to the spectrum of the reference fibre.

Table 2. Radiation-induced attenuation in the $1550 \mathrm{~nm}$ window measured with an OTDR analyser.

\begin{tabular}{lcl}
\hline Fibre sample & Attenuation $\left(\mathrm{dB} \mathrm{km}^{-1}\right)$ & Dose $(\mathrm{MGy})$ \\
\hline Reference & 0.43 & 0 \\
1 & 45 & 0.33 \\
2 & 62 & 0.97 \\
3 & 144 & 4.70 \\
4 & 170 & 9.90 \\
\hline
\end{tabular}

between the two lasers is exactly set at the modulation frequency with no spectral jitter and drift.

Distributed measurements are simply obtained by gating the pump signal through a semiconductor optical amplifier (SOA); pulses are then boosted through an erbium-doped fibre amplifier (EDFA). The probe signal is finally detected by a photodetector and analysed on an oscilloscope.

By varying the frequency of the CW modulation applied to the master output light, the Brillouin gain spectrum of the fibres is completely scanned. The oscilloscope traces are collected and processed in order to retrieve the spectral distribution of Brillouin gain at any position along the fibre, and then to calculate the Brillouin shift and the Brillouin linewidth at the exact location of the irradiation. The injectionlocking technique being intrinsically stable, the Brillouin gain spectrum can be measured with very high accuracy.

\section{Experimental results}

The fibre samples have been tested for 6 weeks after the gamma irradiation and have been monitored over several days.

We first measured the total radiation-induced attenuation in the $1550 \mathrm{~nm}$ window for each sample with a commercial OTDR analyser. The results, summarized in table 2, are comparable with previous works [12].

Figure 2 shows and compares the spectral responses of the radiation-induced absorption measured by the cutback method, using a tungsten-halogen lamp and an optical spectrum analyser. This behaviour is typical of doped optical fibres when exposed to ionizing radiation [12]. However, despite the significant radiation-induced absorption, frequency-based systems-like Brillouin distributed sensors-still operate unbiased since the radiation-induced attenuation only affects the signal-to-noise ratio. 


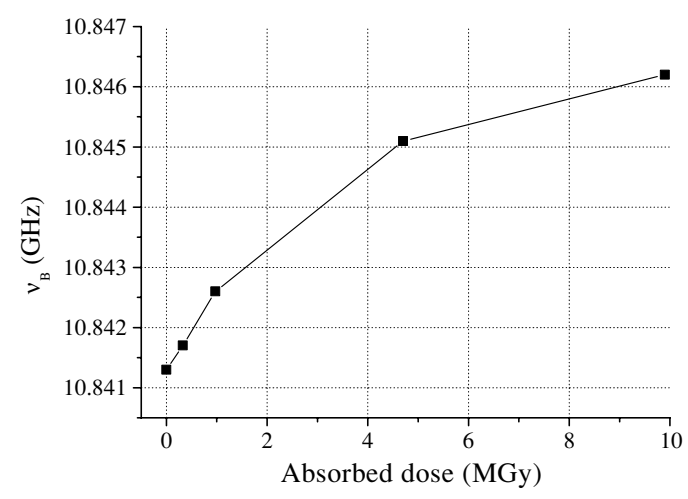

Figure 3. Brillouin central frequency $v_{\mathrm{B}}$ measured as a function of the absorbed dose. All measurements were performed at a constant temperature $(T=295.25 \pm 0.05 \mathrm{~K})$.

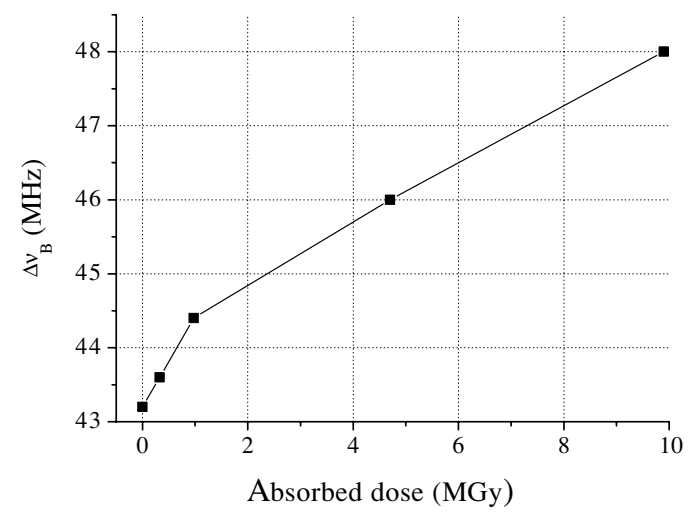

Figure 4. Brillouin FWHM linewidth $\Delta v_{\mathrm{B}}$ measured as a function of the absorbed dose. All measurements were performed at a constant temperature $(T=295.25 \pm 0.05 \mathrm{~K})$.

Table 3. Brillouin frequency and Brillouin linewidth as a function of the absorbed dose. The accuracy of the Brillouin central frequency is $\pm 50 \mathrm{kHz}$ and the accuracy of the half-linewidth is $\pm 200 \mathrm{kHz}$.

\begin{tabular}{llll}
\hline $\begin{array}{l}\text { Fibre } \\
\text { sample }\end{array}$ & $\begin{array}{l}\text { Brillouin } \\
\text { frequency }(\mathrm{GHz})\end{array}$ & $\begin{array}{l}\text { Brillouin } \\
\text { linewidth }(\mathrm{MHz})\end{array}$ & $\begin{array}{l}\text { Dose } \\
(\mathrm{MGy})\end{array}$ \\
\hline Reference & 10.8413 & 43.2 & 0 \\
1 & 10.8417 & 43.6 & 0.33 \\
2 & 10.8426 & 44.4 & 0.97 \\
3 & 10.8451 & 46.0 & 4.7 \\
4 & 10.8462 & 48.0 & 9.9 \\
\hline
\end{tabular}

Figures 3 and 4 show the Brillouin central frequency $\left(v_{\mathrm{B}}\right)$ and the Brillouin linewidth $\left(\Delta \nu_{\mathrm{B}}\right)$ as a function of the absorbed dose. The results presented here were obtained by averaging several measurements carried out under the same conditions and at the same ambient temperature $(T=295.25 \pm 0.05 \mathrm{~K})$, in order to be free of systematic errors which could bias our data.

The results are summarized in table 3 and show a small but clear dependence of the Brillouin scattering on the ionizing radiation: frequency and linewidth increase nonlinearly as a function of the dose.

\section{Discussion}

The capability of Brillouin sensors to measure temperature or strain variations is intrinsically related to the physical origin

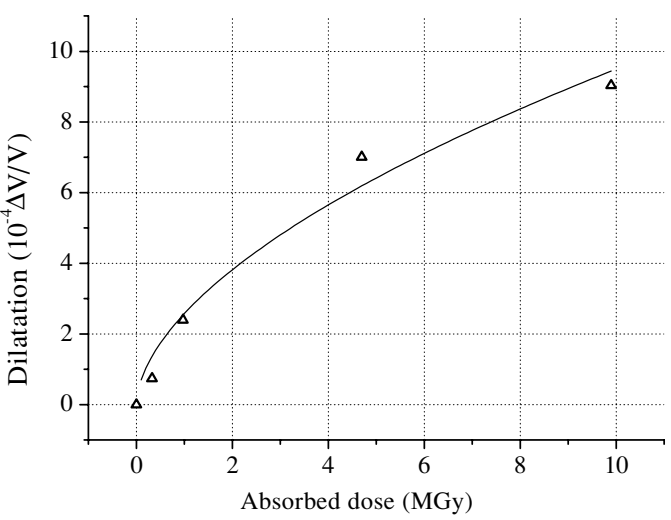

Figure 5. Dilatation due to ionization radiation as a function of the absorbed dose. Experimental data have been fitted with a power law curve $(A=2.577$ and $c=0.566)$.

of the Brillouin scattering, resulting from the change in the acoustic velocity with respect to variations of the silica density. In particular, the acoustic velocity is related to the density by the following equation [13]:

$$
V_{\mathrm{a}}=\sqrt{\frac{E(1-v)}{(1+v)(1-2 v) \rho}}
$$

where $E, v$ and $\rho$ represent respectively the Young modulus, the Poisson number and the density.

Since the Brillouin frequency $v_{\mathrm{B}}$ is related to the acoustic velocity by the relation [14]

$$
v_{\mathrm{B}}=2 n V_{\mathrm{a}} / \lambda_{0}
$$

where $n$ and $\lambda_{0}$ represent respectively the refractive index and the pump wavelength, a variation of the density induces a variation of the Brillouin frequency $v_{\mathrm{B}}$.

Our results show a shift of the Brillouin frequency $v_{\mathrm{B}}$ due to ionization radiation: this tends to indicate a change in the silica density during the irradiation, by means of (4.1) and (4.2). This phenomenon is known as silica compaction and has already been studied in bulk silica [15]. In our case, we observe a negative compaction, i.e. dilatation, due to ionization (see figure 5).

These results confirm the pioneer work of Starodubstev and Azizov [16]. In addition, figure 5 shows that the dose dependence of the dilatation obeys a power law as previously shown [17]:

$$
\frac{\Delta V}{V}=A D^{c}
$$

where $V$ is volume, $D$ is dose and $A$ and $c$ are constants. By fitting our experimental data we have obtained $A=2.577$ and $c=0.566$.

Primak also showed that the sign of the compaction strongly depends not only on the silica type but also on the type and concentration of the dopants $[17,18]$. The radiationinduced compaction being a non-reversible phenomenon (meaning that the shifted Brillouin frequency is then frozen in the fibre, even for discontinued exposure to ionizing radiation) opens the ways for preparing optical fibres with a reduced shifted Brillouin frequency by carefully choosing the dopant concentration and the fibre type, together with pre-irradiation. Measurements of the Brillouin gain spectrum 
done 10 months after the gamma irradiation confirm the nonreversibility of the compaction, the frequencies being the same as those shown in table 3 .

The reason behind the growth of the Brillouin linewidth is not clear and needs further investigations due to the complex interactions of ionizing radiation with silica. This may indicate a structural change of the silica with partial crystallization.. Nevertheless, this modest change causes no impairment for the measurement of the Brillouin frequency $v_{\mathrm{B}}$, which is essential information for sensing.

The frequency variation is about $5 \mathrm{MHz}$ for both frequency and linewidth for the worst case (most irradiated sample), which corresponds approximately to a temperature change of about $5 \mathrm{~K}$ for a total dose of about $10 \mathrm{MGy}$. However, it is important to note that the required radiation acceptance level for nuclear monitoring instrumentation is of the order of 10-100 kGy max. Thus the radiation-induced shift of the Brillouin frequency cannot exceed $0.1 \mathrm{MHz}$ or about $0.1 \mathrm{~K}$ on an equivalent temperature scale, so that it can be considered to be practically negligible in real applications.

\section{Conclusions}

The effect of gamma-radiation on the physical properties of Brillouin scattering in commercially available optical fibres has been investigated up to very high total gamma doses. The frequency shift due to the ionizing radiation is about $5 \mathrm{MHz}$ for the worst case. Distributed sensors based on stimulated Brillouin scattering can thus be considered to be radiationtolerant up to total doses of about $100 \mathrm{kGy}$, provided that the signal-to-noise ratio is kept acceptable. Distributed fibre sensors based on Brillouin spectral analysis can in this way be an efficient monitoring tool for nuclear facilities. Further work on different fibres with different dopant concentration types will indicate which fibre is more suitable for nuclear environments.

In addition, the use of the radiation-induced Brillouin shift as a tool of analysis will certainly bring new perspectives in the understanding of the compaction mechanism in irradiated amorphous silica.

\section{Acknowledgments}

AFF wishes to acknowledge the support of the European Commission under the contract of Association between Euratom and the Belgian State, carried out within the framework of the European Fusion Development Agreement (EFDA). The views and opinions expressed herein do not necessarily reflect those of the European Commission.

\section{References}

[1] Friebele E, Long K, Askins C, Marrone M and Griscom D 1985 Overview of radiation effects in fibre optics, in optical fibre sensing and systems in nuclear environments Proc. SPIE 541 70-88

[2] Berthold J 1994 Overview of prototype fibre optic sensors for future application in nuclear environments, in optical fibre sensing and systems in nuclear environments Proc. SPIE 2425 75-83

[3] Fernandez Fernandez A, Berghmans F, Brichard B and Decréton M 2002 Towards the development of radiation-tolerant instrumentation data links for thermonuclear fusion experiments IEEE Trans. Nucl. Sci. 49 2879-87

[4] Jensen F, Takada E, Nakazawa M, Kakuta T and Yamamoto S 1996 Distributed Raman temperature measurement system for monitoring of nuclear power plant coolant loops Proc. SPIE 2895 132-44

[5] Craik N G 1996 Detection of leaks in steam lines by distributed fibreoptic temperature sensing (DTS) Proc. IAEA Specialists' Meeting Monitoring and Diagnosis Systems to Improve Nuclear Power Plant Reliability and Safety (Gloucester, UK, May)

[6] Kimura A, Takada E, Fujita K, Nakazawa M, Takahashi H and Ichige S 2001 Application of a Raman distributed temperature sensor to the experimental fast reactor JOYO with correction techniques Meas. Sci. Technol. 12 966-73

[7] Fernandez Fernandez A, Brichard B, Berghmans F and Decréton M 2002 Dose-rate dependencies in gamma-irradiated in-fibre Bragg gratings IEEE Trans. Nucl. Sci. 49 2874-8

[8] Fernandez Fernandez A, Ooms H, Brichard B, Coeck M, Coenen S, Berghmans F and Decréton M 2002 SCK • CEN irradiation facilities for radiation tolerance assessment Proc. IEEE NSREC 2002, Radiation Effect Data Workshop (Phoenix, AZ) pp 171-6

[9] Niklès M, Thévenaz L and Robert Ph-A 1996 Simple distributed fibre sensor based on Brillouin gain spectrum analysis Opt. Lett. 21 758-60

[10] Thévenaz L, Le Floch S, Alasia D and Troger J 2004 Novel schemes for optical signal generation using laser injection locking with application to Brillouin sensing, Meas. Sci. Technol. 15 1519-24

[11] Tröger H J 1999 Injection locking in semiconductor lasers, PhD Thesis Ecole Polytechnique Fédérale de Lausanne, EPFL

[12] Ott M 2002 Radiation effects data on commercially available optical fibre: database summary IEEE Nuclear Science and Radiation Effects Conf. (Phoenix, AZ), NSREC 2002, Data Workshop Proceedings

[13] Timoshenko S P and Goodier J N 1970 Theory of Elasticity (New York: McGraw-Hill) chapter 14

[14] Agrawal G P 1989 Nonlinear Fiber Optics (New York: Academic)

[15] Primak W 1975 The compacted states of vitreous silica Studies of Radiation Effects in Solids vol 4 ed G Dienes and L Chadderton (New York: Gordon and Breach)

[16] Starodubstev S V and Azizov S Change in linear dimensions of fused quartz during gamma irradiation Proc. Tashkent Conf. AEC-tr 6398 vol 1 p 283

[17] Ruller J A and Friebele E J 1991 The effect of gamma-irradiation on the density of various types of silica J. Non-Cryst. Solids 136 163-72

[18] Primak W and Edwards E 1962 Radiation-induced dilatations in vitreous silica Phys. Rev. 128 2580-8

[19] Primak W and Kampwirth R 1968 Impurity effect in the ionization dilatation of vitreous silica J. Appl. Phys. 396010 